\title{
Low claudin expression is associated with high Gleason grade in prostate adenocarcinoma
}

\author{
P. VÄRE ${ }^{1}$, I. LOIKKANEN ${ }^{2}$, P. HIRVIKOSKI ${ }^{1}$, M.H. VAARALA ${ }^{2}$ and Y. SOINI ${ }^{1,3}$ \\ ${ }^{1}$ Department of Pathology, University of Oulu and Oulu University Hospital; ${ }^{2}$ Department of Surgery, \\ Division of Urology, Oulu University Hospital, Oulu; ${ }^{3}$ Department of Clinical Pathology \\ and Forensic Medicine, University of Kuopio, Kuopio, Finland
}

Received July 30, 2007; Accepted September 3, 2007

\begin{abstract}
The expression of claudins 1, 2, 3, 4, 5 and 7 was studied in prostate adenocarcinoma and compared to that of non-neoplastic epithelium and Gleason score of the tumors. Additionally, RT-PCR was performed for claudins 2 and 5 in three cases. Strong immunoreactivity of claudins 1, 3, 4 and 7 was seen in prostate adenocarcinoma while expression of claudins 2 and 5 was weaker. In relation to non-neoplastic glands, expression of claudins 2 and 5 was diminished. There was a significant association between the Gleason score and claudin 1 and 5 expression, these claudins were more strongly expressed in tumours with a lower Gleason score. A combined lowered claudin expression was associated with a high Gleason score and a poor prognosis. According to the results, there is a strong claudin expression in prostate adenocarcinoma, especially for claudins 3 and 4 . In contrast, claudin 2 was low in neoplastic cells compared to non-neoplastic epithelium, suggesting downregulation of synthesis or altered metabolism/ assembly of this protein during carcinoma development. The association of lowered claudin 1 and 5 expression and lowered overall claudin expression with tumours of a higher Gleason score suggest that claudins may modulate the histologic features of these tumors and in this way influence their biological behaviour.
\end{abstract}

\section{Introduction}

Prostate cancer is the most frequent malignant tumor in men (1). Most of these tumors are adenocarcinomas and their histological aggressiveness is evaluated by the Gleason grading system where grades are evaluated by changes in the glandular architecture (1). Glandular architecture is partly influenced by cellular polarity and cell to cell contacts and it could thus be hypothesized that changes and dysregulations of proteins mediating cellular contacts might influence the histology and Gleason grade.

Correspondence to: Dr Ylermi Soini, Department of Pathology, University of Oulu, PO Box 5000 (Aapistie 5), 90014 Oulu, Finland E-mail: msoini@cc.oulu.fi

Key words: prostate, cancer, claudin, Gleason score
Claudins are tight junctional proteins, which are present in epithelial and endothelial cells $(2,3)$. They regulate the paracellular permeability of cell layers and serve as a barrier between the apical and basolateral section of the cells thus influencing cell polarity (2-4). Claudins have different properties in their action of regulating paracellular permeability and different claudins may be expressed in different types of cell (2-4). In kidney tubular cells, for example, claudins are expressed in different parts of the segment and during embryonic development their expression may also vary (5).

At least 23 different claudins have been identified (6). Claudins 1, 2, 3, 4 and 7 are widely present in different epithelia while claudin 5 is mainly present in endothelial cells $(2-4,6)$. It participates in the formation of the blood brain barrier (3). Claudin 14 deficiency is associated with hereditary deafness and claudin 16 mutations cause hypomagnesemia $(4,7)$. Claudins may play a role in many diseases in which paracellular efflux of ions and molecules is disturbed such as diseases of the cardiovascular and gastrointestinal tract (4). Clostridium perfringens, for instance, which causes food poisoning, excretes a toxin, and claudins 3 and 4 act as receptors for this toxin, thus contributing to disturbance of the adhesion function of epithelial cells of the intestine and contributing to fluid leakage between paracellular spaces (4).

Claudins are also present in neoplasms originating from epithelial and endothelial cells. Claudins 1, 2, 3, 4 and 7 are abundantly present in different carcinomas, but expression in individual tumours may vary (8). There may also be differences in different histological tumours from the same sites and between tumours of different sites. In gastric carcinoma, for example, expression of many claudins is downregulated in the diffuse subtype compared to the intestinal subtype and in breast carcinoma there are differences in claudin expression between ductal and lobular carcinomas (9-11). In vascular tumours claudin 5 is abundantly present and it can be used as a marker for endothelial differentiation compared to other soft tissue tumours which ordinarily do not express claudin 5 to such an extent (8). Claudin 5 is, however, present also in epithelial tumours and variable expression is found in many types of epithelial cancer (8).

To test the expression of claudins $1,2,3,4,5$ and 7 in prostate adenocarcinoma and to compare the results with Gleason grading and clinical data of the tumors we analysed 
101 cases of prostate adenocarcinoma and compared the results with that of non-neoplastic glands. Additionally RT-PCR for claudins 2 and 5 was performed in three cases.

\section{Materials and methods}

Study materials. The study material consisted of 101 randomly selected prostate tissue samples collected from the archives of the Department of Pathology, University of Oulu and consisted of both electroresection samples or surgical prostatectomy samples. The diagnosis and the Gleason scoring of the cases was based on the WHO classification of urological tumours (1). The Gleason score ranged from $2+2=4$ to $5+5=10$. PSA (prostate specific antigen) levels, taken preoperatively during the same patient visit, were also recorded. The Gleason score was evaluated independently by two investigators with a $\kappa$ coefficient of $0.531(\mathrm{p}<0.001)$ for high $(>7)$ and low $(<7)$ score. Clinical data, such as TNM status and survival data was collected from patient records. The study was approved by the ethics committee of Oulu University Hospital. All samples were fixed in formalin and embedded in paraffin.

Additionally, we collected fresh samples of prostate carcinoma tissue and corresponding non-neoplastic tissue from three prostatectomy cases for mRNA analysis of claudins 2 and 5. After receiving the cases from the operating theatre, histologically confirmed tumor and non-neoplastic tissue samples were frozen in liquid nitrogen in vials containing RNA stabilization mixture (RNA later). Written consent was obtained from every patient giving fresh tissue samples for the study.

Immunohistochemical staining. The primary antibodies used in the immunostaining were all purchased from Zymed Laboratories Inc (South San Fransisco, CA) designed to be used in formalin-fixed paraffin-embedded tissues. They were polyclonal rabbit anti-claudin 1 (clone JAY.8), monoclonal mouse anti-claudin 2 antibody (clone 12H12), polyclonal rabbit anti-claudin 3 (clone Z23.JM), monoclonal mouse anticlaudin 4 (clone 3E2C1), monoclonal mouse anti-claudin 5 (clone 4C3C2) and polyclonal rabbit anti-claudin 7 (clone ZMD.241). Before application of the primary antibodies, the sections were heated in a microwave oven in $10 \mathrm{mM}$ citrate buffer, $\mathrm{pH} 6.0$, for $10 \mathrm{~min}$. After a 60-min incubation with the primary antibody (dilution 1:50 for anti-claudin 1, 2, 3, 4, 5 and 7), a biotinylated secondary anti-rabbit antibody and Histostain-SP kit (Zymed Laboratories Inc) was used. For all the immunostainings, the colour was developed by diaminobenzidine, whereafter the sections were lightly counterstained with hematoxylin and mounted with Eukitt (Kindler, Freiburg, Germany).

Negative control stainings were carried out by substituting non-immune rabbit or mouse serum and PBS for the primary antibodies. As positive controls non-neoplastic kidney, breast, skin and liver samples were used.

The quantity of the immunostaining was assessed by the presence and disruption of the membrane bound immunostaining as follows: $0(-)$, no immunostaining present; 1 (+), $<25 \%$ of cells positive; $2(++), 25-50 \%$ of cells positive; 3 $(+++), 50-75 \%$ of cells positive; and $4(++++), 75-100 \%$ of cells positive.

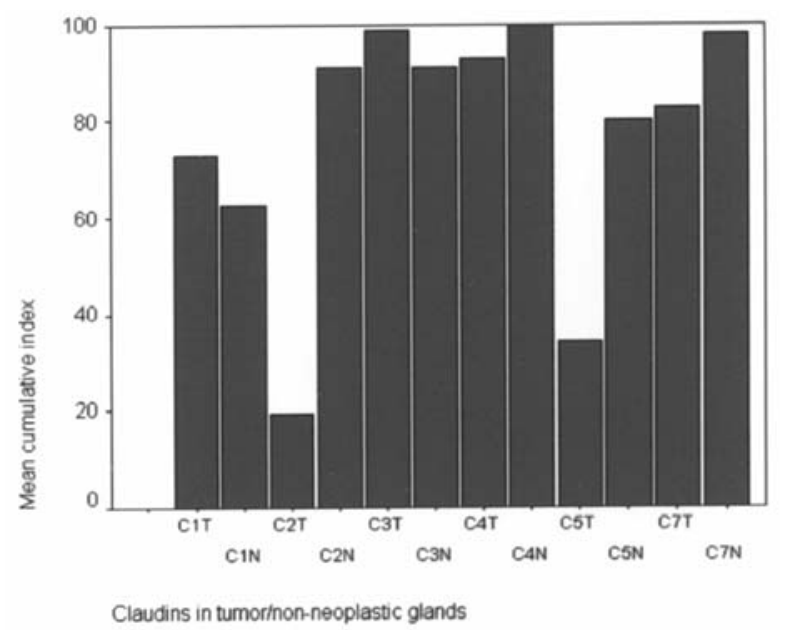

Figure 1. A graph showing claudin expression in tumour and non-neoplastic tissues using a cumulative index. T, tumour; and N, non-neoplastic tissues; and individual claudins with numbers from 1 to 7 . A strong diminution of claudin 2 reactivity in tumor cells compared to non-neoplastic glands (bars 3 and 4 from left, respectively) was observed. Likewise, claudin 5 expression was diminished in tumour tissue (bars 9 and 10 from left) while expression of claudins 3 and 4 was similar in neoplastic and non-neopastic glands (bars 5-8).

Claudin immunoreactivity was assessed separately by the two investigators and the correspondence of the individual results was good $(\kappa$ coefficient $=0.324, \mathrm{p}<0.001$ ) .

A cumulative index for immunoreactivity was obtained by adding together the immunohistochemical staining score for each individual claudin in tumours and in non-neoplastic prostate and comparing this value with the maximal value of the sample set. Cumulative index is given as a percentage of maximal value in Fig. 1.

$R T-P C R$. Total RNA from prostate tissues was isolated using the QuickPrep ${ }^{\text {TM }}$ Total RNA extraction kit (Amersham Biosciences). After the first-strand cDNA synthesis with the high capacity cDNA reverse transcription kit (Applied Biosystems), PCR reaction was performed using Phusion ${ }^{\mathrm{TM}}$ high fidelity DNA polymerase (Finnzymes) and specific primers for claudin 2 and claudin 5 . For claudin 2, the forward primer was TTGTGACAGCAGTTGGCTTC, and the reverse primer was TCAGGGAGAACAGGGAAGAA, with a product size of $408 \mathrm{bp}$. For claudin 5, the forward primer was CTGG ACCACAACATCGTGAC, and the reverse primer was CAG CTCGTACTTCTGCGACA and the product size was $375 \mathrm{bp}$. The PCR conditions were as follows: $98^{\circ} \mathrm{C}$ for $30 \mathrm{sec}$, then 30 cycles of $98^{\circ} \mathrm{C}$ for $10 \mathrm{sec}, 64^{\circ} \mathrm{C}$ for $30 \mathrm{sec}, 72^{\circ} \mathrm{C}$ for 30 sec. The final extension step lasted for $10 \mathrm{~min}$ at $72^{\circ} \mathrm{C}$. PCR products were visualized on $1 \%$ agarose gel. $\beta$-actin RT-PCR was performed simultaneously as an internal control for RNA quality and loading.

Statistical analysis. SPSS for Windows (Chicago, IL) was used for statistical analysis. The significance of associations was determined using Fisher's exact probability test, and two-tailed t-test. Survival analysis was performed using the Kaplan-Meyer method and the associations were determined using log-rank, Breslow and Tarone-Ware tests. 
Table I. Claudin expression in prostate adenocarcinoma and in relation to Gleason score.

\begin{tabular}{|c|c|c|c|c|c|c|c|c|c|c|}
\hline & \multicolumn{5}{|c|}{ Claudin 1} & \multicolumn{5}{|c|}{ Claudin 2} \\
\hline & - & + & ++ & +++ & ++++ & - & + & ++ & +++ & ++++ \\
\hline Gleason $<7$ & 0 & 0 & 7 & 9 & 29 & 14 & 18 & 10 & 3 & 1 \\
\hline \multirow[t]{3}{*}{ Gleason $\geq 7$} & 3 & 11 & 15 & 13 & 14 & 29 & 13 & 7 & 4 & 1 \\
\hline & \multicolumn{5}{|c|}{ Claudin 3} & \multicolumn{5}{|c|}{ Claudin 4} \\
\hline & - & + & ++ & +++ & ++++ & - & + & ++ & +++ & ++++ \\
\hline Gleason $<7$ & 0 & 0 & 0 & 1 & 44 & 0 & 0 & 1 & 3 & 41 \\
\hline \multirow[t]{3}{*}{ Gleason $\geq 7$} & 1 & 1 & 1 & 3 & 50 & 1 & 3 & 2 & 8 & 42 \\
\hline & \multicolumn{5}{|c|}{ Claudin 5} & \multicolumn{5}{|c|}{ Claudin 7} \\
\hline & - & + & ++ & +++ & ++++ & - & + & ++ & +++ & ++++ \\
\hline Gleason $<7$ & 3 & 12 & 14 & 12 & 2 & 0 & 1 & 4 & 8 & 29 \\
\hline Gleason $\geq 7$ & 17 & 15 & 10 & 12 & 2 & 3 & 1 & 3 & 17 & 32 \\
\hline
\end{tabular}

\section{Results}

Non-neoplastic tissue. In non-neoplastic prostatic glands, strong expression with linear membrane bound positivity was found for claudins 2,3,4 and 7. Claudins 5 and 1 showed a slightly weaker positivity. With the scoring system used for tumours, claudins 1, 2, 3, 4, 5 and 7 showed 63, 91, 91, 100,80 and $98 \%$ of the possible maximal value, respectively. No significant difference in staining was observed between hyperplastic or normal prostate glands (data not shown).

Claudins in prostate adenocarcinoma. The results for the tumours are shown in Table I and Fig. 2. Claudin 1 was positive in all but three cases of the 101 adenocarcinomas of the prostate $(97 \%)$. Very strong positivity $(++++)$ was observed in 43 cases (43\%). Compared to non-neoplastic glands, claudin 1 showed a slight increase in the cumulative value.

In contrast to claudin 1, $43(43 \%)$ cases were negative for claudin 2 and there were only two cases which showed strong positivity. The remainder of the cases were weakly (+) or moderately $(++)$ positive. Compared to non-neoplastic glands there was a clear abrogation of claudin 2 positivity.

On the other hand, claudin 3 showed very strong positivity in prostate adenocarcinoma. All but three cases were strongly or very strongly $(+++,++++)$ positive for claudin $3(97 \%)$ and only one case was moderately positive. Non-neoplastic glands were also strongly positive.

Claudin 4 showed also strong positivity in prostate adenocarcinoma. All but one case was positive of which $83(82 \%)$ were very strongly positive. Normal glands were also strongly positive.

There were 20 cases negative for claudin 5 (20\%). Most of the cases were weakly $(+)$ or moderately $(++)$ positive. Only four cases with very strong positivity $(++++)$ were found. Claudin 5 was more often expressed in normal than in neoplastic tissue.
Claudin 7 was positive in all but three cases (97\%). Sixtyone cases $(62 \%)$ were very strongly positive. According to the cumulative index, expression of claudin 7 was slightly diminished compared to normal glands.

Claudins and Gleason score in prostate adenocarcinoma. The association of claudin immunoreactivity with Gleason scoring is shown in Table I. Cases where the Gleason score was $<7$ showed significantly more cases with moderate, strong or very strong positivity for claudin $1(\mathrm{p}=0.0002)$ and claudin 5 $(\mathrm{p}=0.02)$. In addition, there were significantly more negative cases for claudin 2 in the group with a high Gleason score than in the low Gleason score group ( $\mathrm{p}=0.02$, 29/54 vs 14/46 cases). In a similar manner, there were more cases showing very strong positivity (++++) for claudin 4 in the group with a lower Gleason score than in the group with a higher Gleason score ( $\mathrm{p}=0.03,41 / 45$ vs $42 / 56$ cases). A similar tendency was seen for claudin 3 , but the association was not statistically significant $(\mathrm{p}=0.09)$ and the number of the cases showing less than very strong positivity was, of course, very small in this group.

Additionally, if the scores of all claudins were added together, those cases showing a lower total claudin expression (had a total immunohistochemical score of $<17$ ) had significantly more cases of higher Gleason score $(\mathrm{p}=0.004)$ (Table II).

Other parameters. Mean PSA value in the material was $56.9 \pm 83.7 \mathrm{ng} / \mathrm{ml}$. Cases showing a lower Gleason score had a significantly lower PSA value $(25.3 \pm 24.6 \mathrm{ng} / \mathrm{ml}, \mathrm{p}=0.009)$ than cases with a higher Gleason score $(94.3 \pm 110.8 \mathrm{ng} / \mathrm{ml})$. We found no association between expression of any of the claudins and PSA. However, cases showing a low overall expression of claudins (had a total immunohistochemical score of <17) showed a higher serum PSA value $(83.3 \pm 87.3 \mathrm{ng} / \mathrm{ml}$ vs $32.9 \pm 41.1 \mathrm{ng} / \mathrm{ml}, \mathrm{p}=0.01$ ) (Fig. 3). 

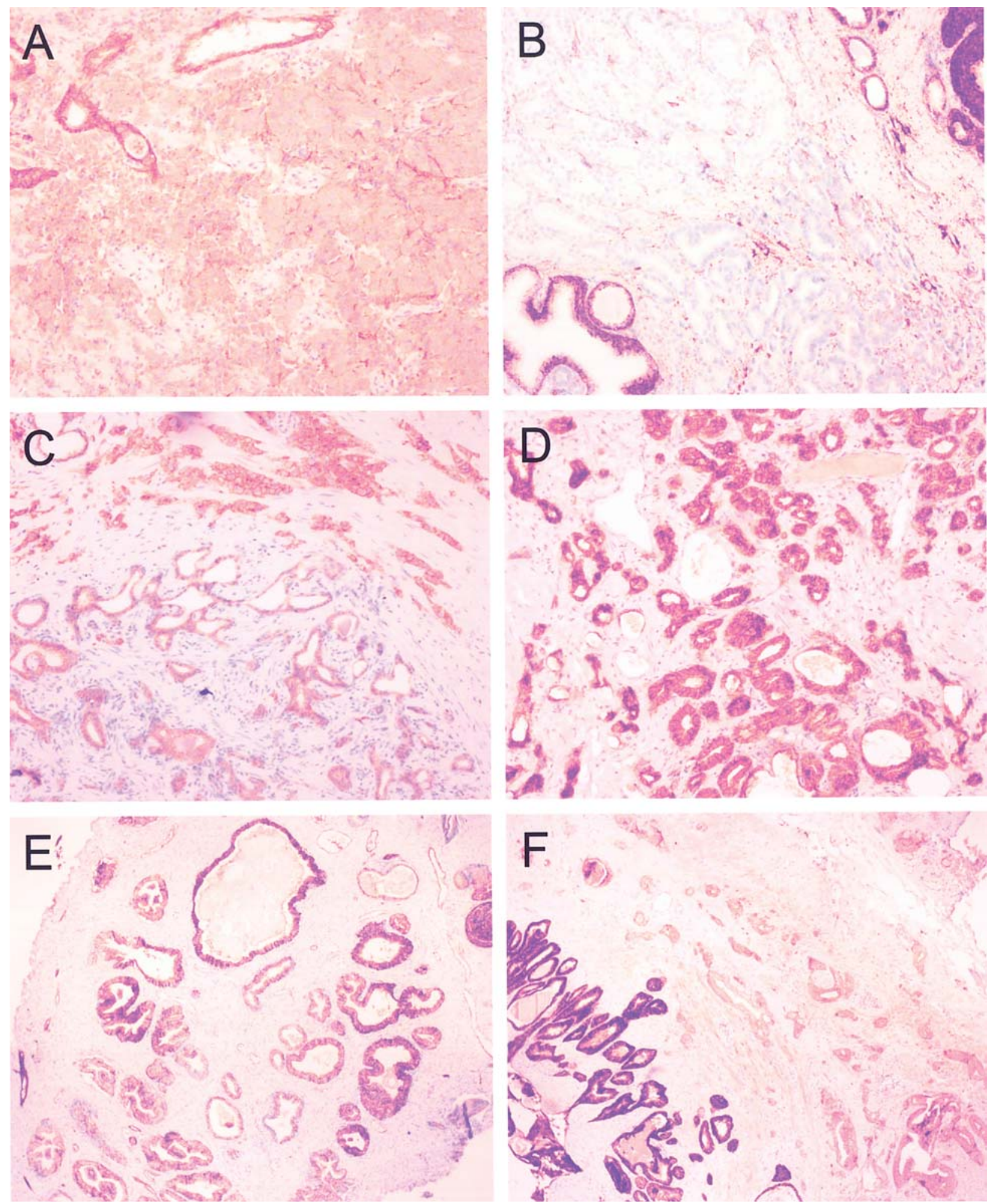

Figure 2. (A) In this case of a prostate adenocarcinoma, claudin 1 was only weakly (+) positive. Non-neoplastic glands (left upper corner), however, showed strong positivity. (B) A prostate adenocarcinoma negative for claudin 2. Non-neoplastic glands (left lower and right upper corner) were strongly positive. (C) Claudin 3 immunoreactivity in prostate adenocarcinoma. Tumor cells (upper) showed strong positivity while a benign hyperplastic nodule (lower) expressed weaker positivity. (D) Strong claudin 4 positivity in a case of prostate adenocarcinoma. (E) Benign glands in prostate tissue showing strong positivity for claudin 5. Nearby blood vessels were also positive. (F) Tumour cells showing weak positivity for claudin 7 (the middle field). Nerve invasion was also observed (the middle field). Benign glands (left corner) showed strong positivity.

In cases where TNM status was known $(n=46)$ there were significantly more cases with a lowered claudin $1(\mathrm{p}=0.04)$ and claudin $5(\mathrm{p}=0.04)$ expression in locally advanced tumors (T3-4) and such a tendency was seen also for claudin 7 $(\mathrm{p}=0.06)$. The metastatic status did not associate with claudin expression. There were more cases with a high Gleason score in the T3-T4 group than in the T1-T2 group $(\mathrm{p}=0.002)$. In survival analysis, individual claudins did not associate with 
Table II. Gleason score in association with total immunoreactivity for claudins.

\begin{tabular}{lccc}
\hline $\begin{array}{l}\text { Total immunoreactivity } \\
\text { for claudins }\end{array}$ & $\begin{array}{c}\text { Gleason } \\
\text { score }<7\end{array}$ & $\begin{array}{c}\text { Gleason } \\
\text { score } \geq 7\end{array}$ & Total \\
\hline$<17$ & 15 & 24 & 39 \\
$\geq 17$ & 37 & 17 & 54 \\
Total & 52 & 41 & 93 \\
\hline
\end{tabular}

$\mathrm{P}=0.004$, Fisher's exact test.

A

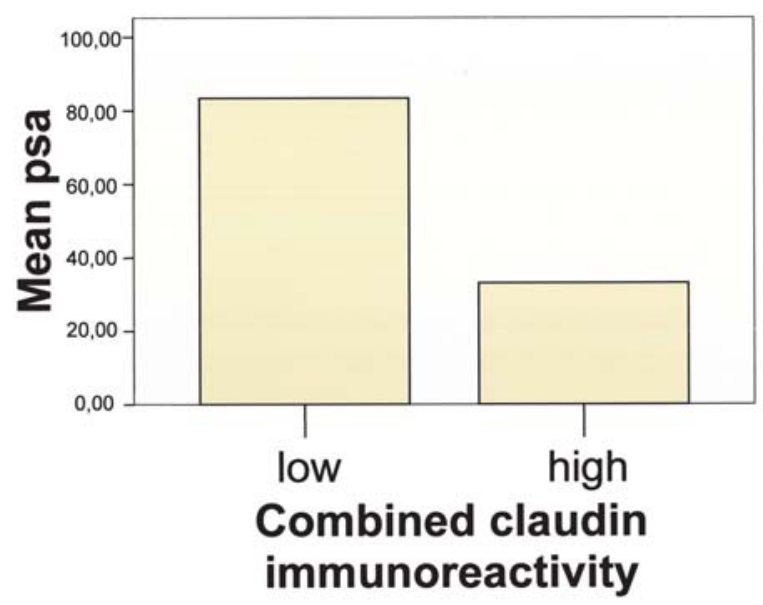

B

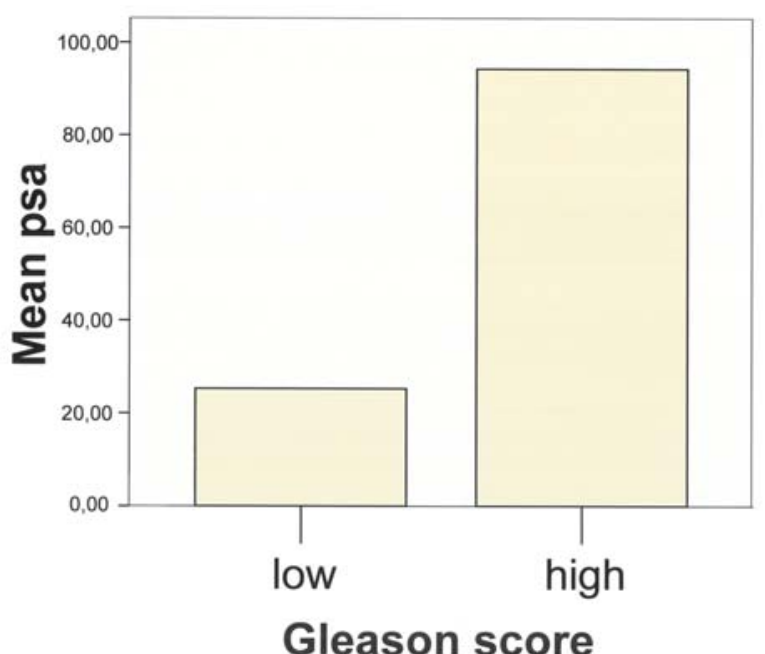

Figure 3. PSA value in relation to combined claudin immunoreactivity or high Gleason score in prostate adenocarcinoma. The sera of patients with tumors with low combined claudin immunoreactivity (A) or with a high Gleason score (B) had a higher PSA value.

patient survival, however, cases showing a higher expression of all claudins (total immunohistochemical index $>17$ ) had a better prognosis $(\mathrm{p}<0.001, \log$-rank, Breslow, Tarone-Ware) (Fig. 4A). Also cases with a lower Gleason score had a better prognosis $(\mathrm{p}<0.001, \log$-rank, Breslow, Tarone-Ware) (Fig. 4B). In Cox multivariate analysis, total expression of
A

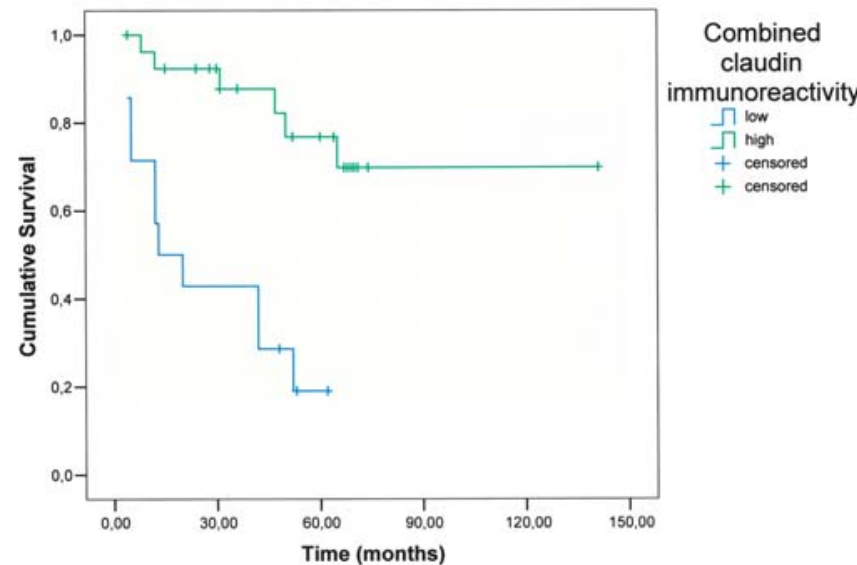

$\mathbf{B}$

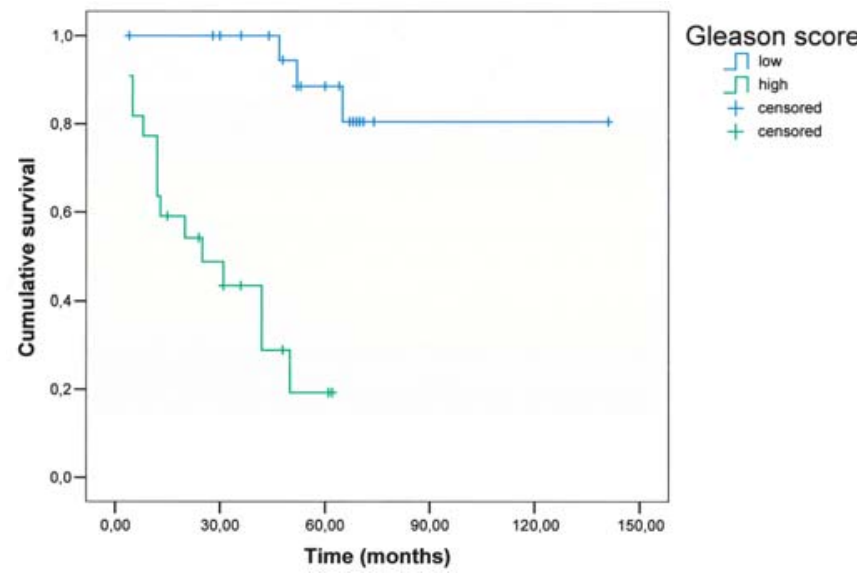

Figure 4. Patient prognosis was worse in cases with a low combined claudin immunoreactivity (A) or a high Gleason score (B).

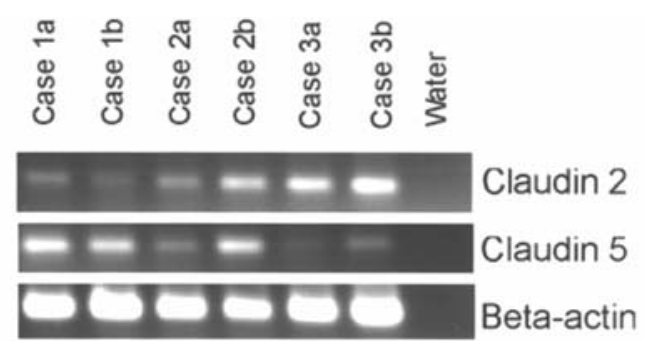

Figure 5. RT-PCR analysis for claudin 2 and 5 mRNA in three cases of tumor and non-neoplastic prostatic tissue. In tumor samples bands for claudin 2 mRNA were detected in all and claudin 5 mRNA in all but one sample while non-neoplastic tissues showed expression in all samples. The $ß$-actin control is shown at the bottom.

claudin did not have independent prognostic value $(\mathrm{p}=0.414)$ while the Gleason score did $(\mathrm{p}=0.003)$.

RT-PCR for claudins 2 and 5. Three cases of prostate carcinoma and corresponding tissue from non-neoplastic prostate were analysed by RT-PCR for claudins 2 and 5 . The results are shown in Fig. 5. Of these case 1 and 2 were immunohistochemically positive for claudin $2(+,+++)$ and $5(+++,+)$ and case 3 was negative for both. All cases showed detectable mRNA bands for claudin 2 and 5 both in tumor and 
non-neoplastic samples but the intensity of the bands varied from case to case.

\section{Discussion}

In this study we investigated the expression of claudins 1, 2, 3, 4, 5 and 7 in 101 prostatic adenocarcinomas and compared the results with normal and hyperplastic prostatic glands present in adjacent sections. According to the results, most frequent expression was found for claudins 3 and 4, but also claudins 1 and 7 showed frequent positivity. The positivity was less frequent for claudin 5 and for claudin 2 , for the latter only two cases showed very strong positivity and 43 cases were negative.

Claudins 3 and 4 have been, in addition to being components of tight junction and controlling paracellular permeability, found to function as receptors for clostridium perfringens toxin, and in cell lines, administration of the toxin to prostatic adenocarcinoma cells leads to apoptosis and necrosis of the cells (12). According to the results presented here, prostate adenocarcinoma cells strongly express both claudin 3 and 4 . Their expression has also been found in other types of epithelial tumours, such as breast, gastric, and pancreatic carcinomas (8-11). Thus, strong expression of these claudins appears to be a rather uniform feature in epithelial neoplasms.

On the other hand, in our analysis of immunohistochemical claudin expression, claudin 2 was low in adenocarcinoma compared to non-neoplastic prostate tissues. The results thus indicate either lowered claudin 2 synthesis, increased degradation or deranged assembly of this protein onto the cell membranes in carcinoma cells. In addition to claudin 2, downregulation of claudins 1 and 5 was also observed. In the RT-PCR analysis for claudin $5 \mathrm{mRNA}$, all three cases showed mRNA to some extent, indicating a synthetic activity even though one carcinoma case was immunohistochemically negative. Claudin 2 mRNA synthesis was also found in all three carcinoma cases. RT-PCR does not, however, give a reliable assessment of the quantity of mRNA synthesis, but indicates that mRNA synthesis is present at least to some degree in these samples.

In our study we compared claudin expression with the Gleason score of the tumours. In our analysis, a strong association was found between low-grade tumours and expression of claudins 1 and 5. In a similar manner, there were less cases showing very strong claudin 4 positivity in the high score Gleason group than in the low score group. Since Gleason score principally associates with the degree of differentiation and glandular formation of tumors, the results are in parallel with the loss of glandular formation and organisation of the malignant epithelial tissue. Indirectly it suggests that, in malignant cells, the frequency or organisation of tight junctional structures would be diminished. Alternatively, synthesis of such claudins might be abrogated or perhaps expression or activation of MMP might be stronger in tumors with a higher Gleason score. MMPs have been shown to be able to degrade some claudins and occludin and, on the other hand, some claudins, such as claudin 3, 4 and 5 have been shown to be able to regulate or activate MMP2 (13-15). Indeed, a higher expression of MMPs has been observed in tumors with a higher Gleason score (16).
We observed a diminished claudin 1 and 5 expression in T3-T4 tumors. However, we also found an association between high Gleason score and T stage, suggesting that the two may be linked. Claudins 1 and 5 may in this way indirectly influence tumor progression. They did not associate, however, with the metastatic status of the tumors. No individual claudin was associated with survival of the patients. However, a high total expression of claudins was associated with a better survival. Again, a favourable prognosis was also associated with a lower Gleason score.

It has also been suggested that claudin 7 might regulate the expression of PSA (17). In our analysis we did not find any association between the PSA level and claudin 7 or any other individual claudin, suggesting that the influence of claudin 7 is at least not so strong that it can be detected in material such as that which we used. On the other hand, cases with an overall lower expression of claudins, however, showed significantly higher PSA values and similarly, cases with a high Gleason score had higher PSA values. It may thus be that a lower concentration of claudins in prostate adenocarcinoma cells causes the tumor cells to leak more, thus contributing to higher PSA values in such cases.

In conclusion, our results show a strong expression of claudins 3 and 4 in prostate adenocarcinoma. In contrast, expression of claudins 2 and 5 is diminished compared to that in non-neoplastic prostate epithelial cells. Expression of claudins 1,4 and 5 is associated with better differentiation and lower Gleason score of the tumors. Diminished total expression of claudins was associated with a low Gleason score and both were associated to a worse patient prognosis. The data suggest that claudins by putatively influencing tumor cell architecture and Gleason score might affect the biological behaviour of prostate adenocarcinoma.

\section{Acknowledgements}

This study was financially supported by the Foundation of the Finnish Anti-Tuberculosis Association and the Regional Cancer Society of Northern Finland.

\section{References}

1. Eble JN, Sauter G, Epstein JI and Sesterhenn IA: Tumours of the Urinary System and Male Genital Organs. In: World Health Organisation Classification of Tumours. Pathology and Genetics. IARC Press, Lyon, France, 2004.

2. Furuse M, Fujita K, Hiiragi T, Fujimoto K and Tsukita S: Claudin 1 and 2; novel integral membrane proteins localizing at tight junctions with no sequence similarity to occluding. J Cell Biol 141: 1539-1550, 1998.

3. Tsukita S and Furuse M: Claudin-based barrier in simple and stratified cellular sheets. Curr Opin Cell Biol 14: 531-536, 2002.

4. Sawada N, Murata M, Kikuchi K, Osanai M, Tobioka H, Kojima T and Chiba H: Tight junctions and human disease. Med Electron Microsc 36: 147-156, 2003.

5. Reyes J, Lamas M, Martin D, del Carmen Namorado M, Islas S, Luna J, Tauc M and Gonzales-Mariscal L: The renal segmental distribution of claudins changes with development. Kidney Int 62: 476-487, 2002.

6. Turksen K and Troy T-C: Barriers built on claudins. J Cell Science 117: 2435-2447, 2004.

7. Wilcox ER, Burton QL, Naz S, Riazuddin S, Smith TN, Ploplis B, Belyantseva I, Ben-Yosef T, Liburn NA, Morell RJ, Kachar B, Wu DK, Griffith AJ, Riazuddin S and Friedman TB: Mutations in the gene encoding tight junction claudin-14 cause autosomal recessive deafness DFNB29. Cell 104: 165-172, 2001. 
8. Soini Y: Expression of claudins 1, 2, 3, 4, 5 and 7 in various types of tumours. Histopathology 46: 551-560, 2005.

9. Soini Y, Tommola S, Helin H and Martikainen P: Claudins 1, 3,4 , and 5 in gastric carcinoma, loss of claudin expression associates with the diffuse subtype. Virchows Arch 12: 1-7, 2005.

10. Resnick MD, Gavilanez M, Newton E, Konkin T, Bhattacharya B, Britt DE, Sabo E and Moss SF: Claudin expression in gastric adenocarcinomas: a tissue microarray study with prognostic correlation. Hum Pathol 36: 886-892, 2005.

11. Soini Y: Claudins 2, 3, 4 and 5 in Paget's disease and breast carcinoma. Hum Pathol 35: 1531-1536, 2004.

12. Long H, Crean CD, Lee W-H, Cummings OW and Gabig TG: Expression of clostridium perfringens enterotoxin receptors claudin 3 and claudin 4 in prostate cancer epithelium. Cancer Res 61: 7878-7881, 2001.
13. Giebel SJ, Menicucci G, McGuire PG and Das A: Matrix metalloproteinases in early diabetic retinopathy and their role in alteration of the blood-retinal barrier. Lab Invest 85: 597-607, 2005.

14. Agarwal R, D'Souza T and Morin PJ: Claudin 3 and claudin 4 expression in ovarian epithelial cells enhances invasion and is associated with increased matrix metalloproteinase- 2 activity. Cancer Res 65: 7378-7385, 2005.

15. Miyamori H, Takino T, Kobayashi Y, Tokai H, Itoh Y, Seiki M and Sato $\mathrm{H}$ : Claudin promotes activation of pro-matrix metalloproteinase-2 mediated by membrane-type matrix metalloproteinases. J Biol Chem 276: 28204-28211, 2001.

16. Stearns $M$ and Stearns ME: Evidence for increased activated metalloproteinase 2 (MMP2a) expression associated with human prostate cancer progression. Oncol Res 8: 69-75, 1996.

17. Zheng JY, Yu D, Foroohar M, Ko E, Chan J, Kim N, Chiu R and Pang S: Regulation of the expression of the prostate specific antigen by claudin 7. Membr Biol 194: 187-197, 2003. 